This item was submitted to Loughborough's Research Repository by the author.

Items in Figshare are protected by copyright, with all rights reserved, unless otherwise indicated.

\title{
Bone fracture monitoring using implanted antennas in the radius tibia and phalange heterogeneous bone phantoms
}

PLEASE CITE THE PUBLISHED VERSION

https://doi.org/10.1088/2057-1976/aab974

PUBLISHER

(C) IOP Publishing Ltd.

\section{VERSION}

AM (Accepted Manuscript)

\section{LICENCE}

CC BY-NC-ND 4.0

\section{REPOSITORY RECORD}

Symeonidis, Simon, William Whittow, Chinthana Panagamuwa, and Massimiliano Zecca. 2018. "Bone Fracture Monitoring Using Implanted Antennas in the Radius Tibia and Phalange Heterogeneous Bone Phantoms". Loughborough University. https://hdl.handle.net/2134/33008. 


\title{
Bone fracture monitoring using implanted antennas in the radius, tibia and phalange heterogeneous bone phantoms
}

\author{
Symeon Symeonidis' ${ }^{1}$, William G. Whittow ${ }^{1}$, Massimiliano Zecca ${ }^{1,2}$, and Chinthana \\ Panagamuwa $^{1}$
}

1. Wolfson School of Mechanical, Electrical and Manufacturing Engineering, Loughborough University, Loughborough, Leicestershire, UK

2. National Centre for Sport and Exercise Medicine (NCSEM), Loughborough University, Loughborough, Leicestershire, UK

$\underline{\text { S.Symeonidis@lboro.ac.uk, w.g.whittow@lboro.ac.uk, m.zecca@lboro.ac.uk, c.j.panagamuwa@lboro.ac.uk }}$

\begin{abstract}
This paper proposes an innovative technique for monitoring the healing of severe bone fractures using a pair of radio frequency monopoles implanted in the fractured bone. It is envisaged that the screws and external fixation used to stabilise and align the bone will also act as monopole antennas. The proposed antenna configurations were measured inside three novel heterogeneous bone phantoms representing the radius, tibia and phalange bones, and the results were compared to computer simulations with a voxel model of a 26-year-old female from CST Microwave Studio's Virtual Family. The power transmitted from one monopole to the other $\left(S_{21}\right)$ was measured and evaluated as an indicator for fracture healing as blood emulating liquid was injected inside the phantom emulating the conditions of a bone fracture. Finally, for the validation of the phantom measurements, an ex-vivo measurement was conducted using a lamb femur bone. In all cases, the power transmitted from one monopole to the other through the fracture decreased significantly as the volume of the blood representing the fracture increased. The proposed system could provide doctors with a quantitative monitoring tool regarding the healing progress of a fractured bone through the reduction of the wound's hematoma during the first four weeks after the trauma that are critical for bone restoration.
\end{abstract}

\section{Introduction}

During the past decade, communications-enabled implanted devices for medical applications have been a growing field of research. Due to the limited space inside the body, the lossy nature of tissues and the obligatory biocompatibility of the implants, designing and manufacturing these devices is extremely challenging. Radio frequency (RF) medical applications presented in the literature mainly operate in the Medical Device Radiocommunications Service (MedRadio) at $401-406 \mathrm{MHz}$ band [1]-[3] or the Industrial, Scientific and Medical (ISM) band at 2.44 to $2.46 \mathrm{GHz}$ [4]-[6]. The location of implanted antennas is usually under the skin or in fat and muscle tissues [7]-[9]. Applications include implants beneath the skull for the transmission of RF signals to antennas out of the body [10], intraocular for retinal prosthesis [11], ingestible capsules for monitoring human intestines [12], and induction loops for the powering sensors and implants inside the human body [13]. 


\subsection{Fracture monitoring}

The bone fracture monitoring period is from the point of surgery following trauma to the point of bone unification. As stated in [14], "In patho-physiology, bone union is achieved when sufficient tensile strength across the fracture is observed". This occurs when the blood coagulates to form periosteal and endosteal calluses in the fractured area that after 5-10 days transform to intermediate callus. Intermediate callus is more mineralized than periosteal and endosteal calluses [15]. Therefore, successful bone union is directly dependent on the progression of the fracture from the initial hematoma to periosteal - endosteal calluses, to intermediate callus and finally to bone. Thus, the progress of bone healing is indicated by the reduction of blood through transformation into the foretold callus stages inside the fracture [16]. This healing process can be affected by the age, nutrition and medical condition of the patient, the size, position and number of discrete fractures, and the amount of stress that is applied during the healing period [16, 21]. These factors may lead to delayed bone healing, a condition where the intermediate callus will not form into healthy bone tissue, leading to severe pain and loss of mobility and muscle strength [20]. Regular monitoring is necessary for sufficient evaluation and treatment of these fractures, as correct bone restoration is highly dependent on the first four weeks of healing and can be difficult to achieve with patients that have surgically implanted metal plates [21]. The available techniques found in literature for the monitoring of fracture healing include MRI, X-rays and CT scanning, which can be costly and can only be applied if the implanted fixator is non-ferromagnetic [22]. With X-rays, regular monitoring results in high levels of ionizing radiation exposure [23], and are not suitable for children and pregnant women.

Several researchers have proposed the use of AC current at lower frequencies as a monitoring technique. The current is conducted through two additional implanted pins to measure the variations of the fracture's impedance as a probable healing indicator. An increase in the impedance of the bone fracture measured at $100 \mathrm{~Hz}$ during the first 4 weeks after the trauma was reported in [24]. Subsequently, the impedance decreased gradually after the fourth week as the bone was restored. Similar results can be found in [23] for measurements at 50kHz in a buffalo joint where it was found that the impedance of the bone was proportional to the size of the fracture that was introduced. Finally in [25], the impedance at $2 \mathrm{~Hz}$ of the fractured bone of a rabbit began increasing from the second week after the fracture when the mineralization process of the bone started.

The major drawback of this technique is that the impedance change is often very small and difficult to distinguish. In our work, a novel approach using the existing pins of an external metal fixator plate as monopoles at the frequency spectrum of 1 to $4 \mathrm{GHz}$ is proposed. Using existing fixators eliminates the need for any additional medical procedures.

According to the literature, the electromagnetic-related properties of bones that have been studied are: capacitance [26], inductance [27], impedance [28], dielectric constant [29] and conductance [30]. It is proposed by the authors to make use of these properties to monitor bone fracture healing. It is expected that the change of the dielectric and conductive properties of the fractured area, due to the reduction of blood as the trauma heals, will have a clearly observable effect on the power transferred from one monopole to the other. Due to the unpredictable geometry of a bone fracture, thus the unpredictable volume of the hematoma, we hypothesize that the magnitude and rate of change over time of the power transmitted between the two monopoles will correlate to the success 
(or failure) of the healing process. As long as, in the investigated fractured area, the amount of blood-hematoma reduces by gradually transforming into callus, the power transferred from one monopole to the other will increase in magnitude. This will be observed as an improvement in $S_{21}$, i.e. the $S_{21}$ becomes closer to $0 \mathrm{~dB}$.

This paper presents an innovative technique for monitoring the healing of severe bone fractures using a pair of radio frequency monopoles to be implanted in the fractured bone. Section 2 describes the two monopole system used for the evaluation of the bone healing process. In Section 2.1 the two monopole system inside a voxel model is simulated and analysed; in Section 2.2 the recipes used for the development of each tissue of the in-house developed multi-material phantoms are presented; and in Section 2.3 the phantom test-beds are reported. Results are presented and discussed in Section 3, followed by the concluding remarks of this work in Section 4.

\section{Materials and experimental methods}

The proposed fracture monitoring system consists of two monopole antennas and it is envisioned to be a part of a commercially available external medical metal plate. The conducting parts of the monopoles represent the medical screws that are implanted perpendicular to the bone either side of the fracture in order to provide support and positioning of bone fragments. The power transferred from monopole 1 to monopole 2 in terms of $S_{21}$ magnitude was investigated as an indicator of the bone's unification - healing progress. According to Gabriel et al. [31], blood's relative permittivity and conductivity are significantly higher compared to bone's across the radiofrequency spectrum. Therefore, as the amount of blood inside and around the fracture decreases in volume, the losses in the propagation medium are also decreased, thus, the magnitude of the $S_{21}$ is expected to increase.

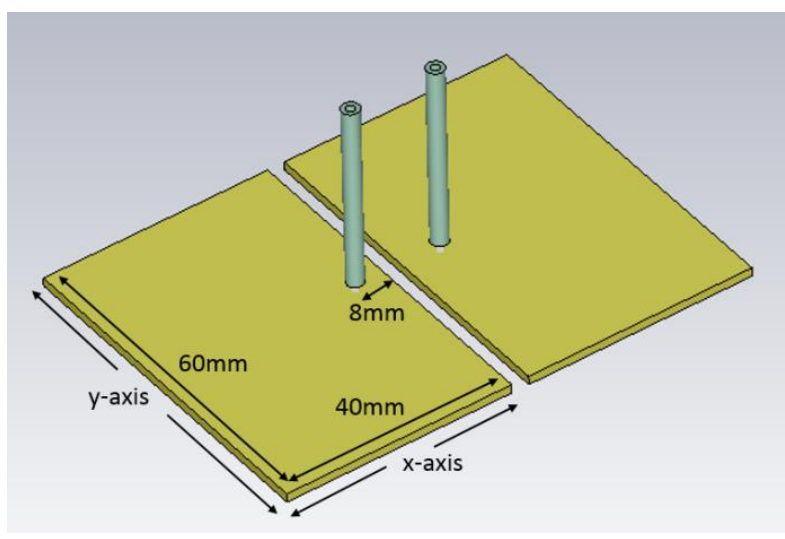

(a)

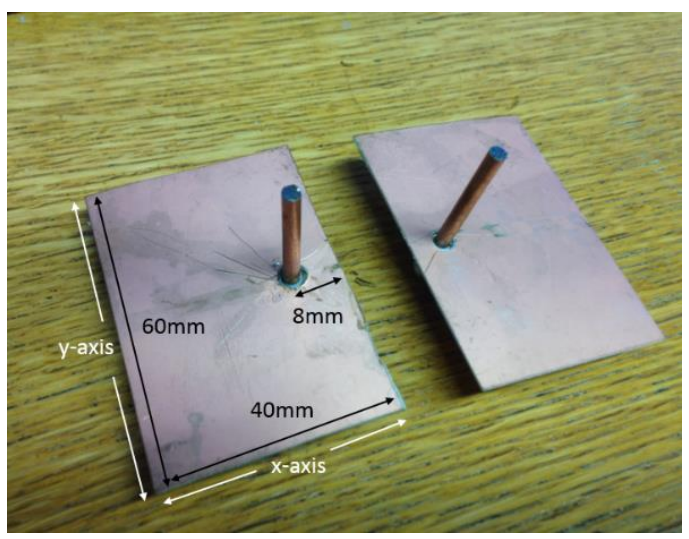

(b)

Figure 1: The monopole antennas of the monitoring system: a) simulated b) fabricated

Two identical monopole antennas were simulated and constructed as shown in Figure 1. The conducting part of the monopoles had a $2.5 \mathrm{~mm}$ diameter and were covered using a cylindrical 3D printed biodegradable plastic polymer (PLA) insulators of $4 \mathrm{~mm}$ outer diameter. The diameter of each coated monopole was comparable to the average fixator screw diameter found in [32]. For the Tibia and Phalange fracture measurements, the monopole 
lengths were $25 \mathrm{~mm}$ and the groundplanes were $60 \times 40 \mathrm{~mm}$. Due to the depth of the radius bone beneath the skin, a second pair of $40 \mathrm{~mm}$ length monopoles with $60 \times 56 \mathrm{~mm}$ groundplanes were also constructed. $5 \mathrm{~mm}$ of the length of each monopole was in free space connected to each groundplane and the rest of their length was inside the body placed through the muscle and perpendicular to the bone. The gap of $5 \mathrm{~mm}$ of free space between the muscle tissue and the groundplanes was used to avoid the groundplanes contacting with the highly conductive muscle layer. The dimensions given to the groundplanes ensured good support of the cylindrical part of the antennas in parallel position along the bone axis while providing acceptable propagation efficiency of the monopoles. A gap of $4 \mathrm{~mm}$ between the groundplanes was selected as adequate space for the injection of blood emulating liquid that was used to emulate the fracture in the measurements section. The distance between the monopoles for all simulations and measurements was $20 \mathrm{~mm}$ as it was found in previous research to be the optimal distance within the fracture providing a good ratio of distance versus wave attenuation [19]. To maintain the $20 \mathrm{~mm}$ distance of the monopoles and the $4 \mathrm{~mm}$ gap between the groundplanes, the conducting parts of the monopoles were attached to their groundplanes at an $8 \mathrm{~mm}$ distance from the middle of the y-axis of the groundplanes (see Figure 1).

\subsection{Simulations using an anatomical human model}

For the simulations of the antenna system, the Voxel model of a 26 year old female of 58kg weight and $1.60 \mathrm{~m}$ height from the CST Studio Virtual Family of the was used [33]. The permittivity and conductivity of the model's tissues were frequency dependent. Their values changed for each simulated frequency according to Gabriel's measurement results found in [31] for the range of 1 to $4 \mathrm{GHz}$. The representation of the fracture was envisioned by two coaxial cylinders. The inner cylinder of the fracture was placed in the $20 \mathrm{~mm}$ mid distance between the two monopoles and replaced the bone in that area. The diameter of the cylinder was equal to the diameter of the investigated bone and its length was set at $16 \mathrm{~mm}$. For the replication of bone healing, the dielectric properties given to the inner cylinder shifted from blood towards bone for each simulation. The outer cylinder surrounding the bone and was given the dielectric properties of blood as it represents the hematoma around the bone fracture [34]. At the initial fractured state, the maximum amount of hematoma was simulated. The length and radius of the outer cylinder were $50 \mathrm{~mm}$ and $15.5 \mathrm{~mm}$ respectively for the radius model, for $30 \mathrm{ml}$ of blood hematoma (see Figure 2). At the final simulation, the fracture was considered healed and the inner cylinder had the dielectric properties of the bone and the outer cylinder was removed from the simulation. 


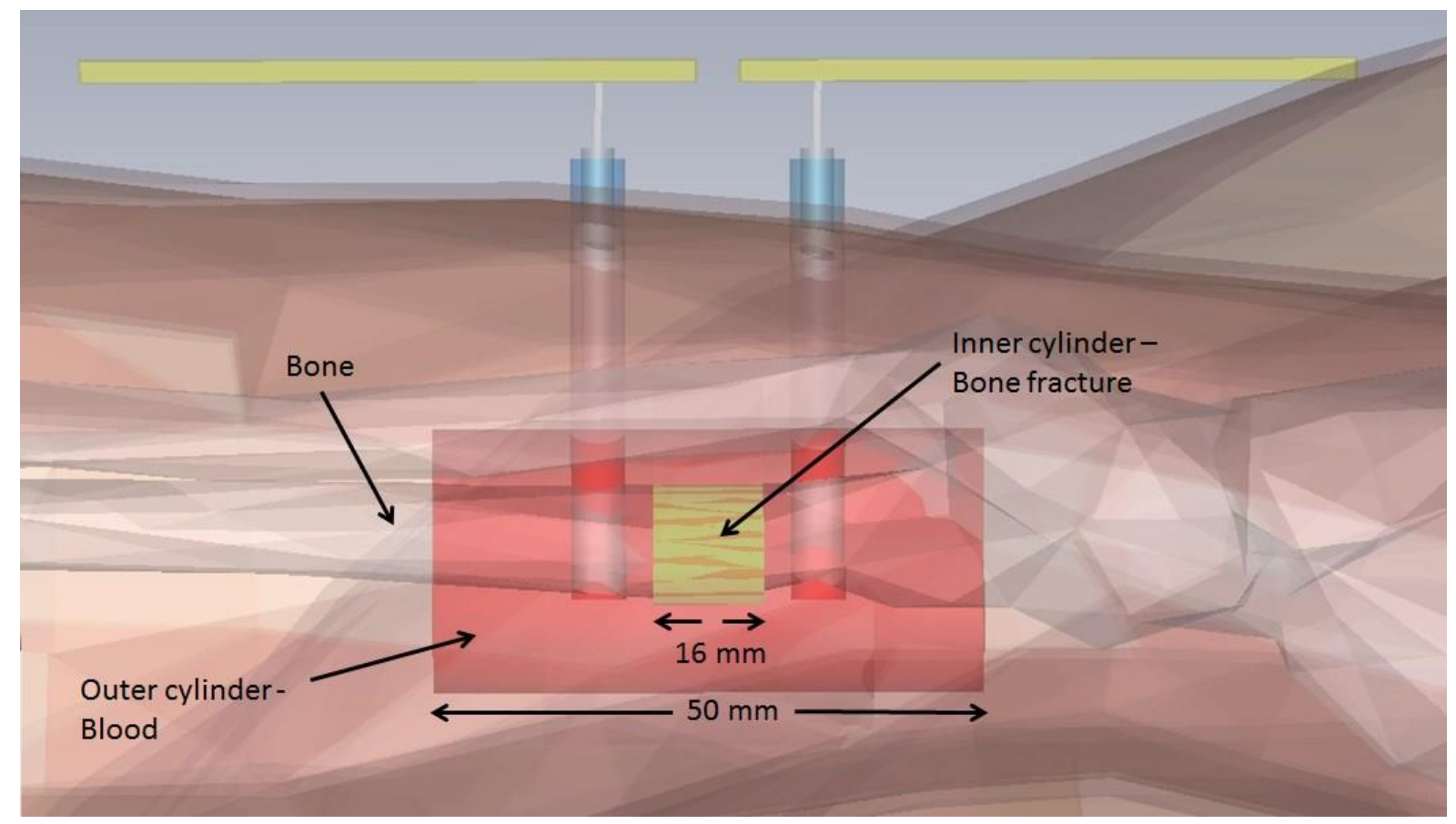

Figure 2: Simulation of the bone fracture using two coaxial cylinders inside a Voxel model

\subsection{Development of homogenous tissue-mimicking phantom recipes}

In order to conduct practical testing, an in-house tissue mimicking phantom was developed. The amounts of each of the materials that were used can be seen in Table 1. The process followed for producing the materials with equivalent dielectric properties to actual tissue, has been described in detail by the authors in [35]-[37]. For the measurements of the dielectric properties of phantom bone cortical, blood and muscle, an open-ended RG-402 semi-rigid coaxial cable - probe was used over the 0.5 to $4 \mathrm{GHz}$ frequency range $[38,39]$.

Table 1: Tissue-mimicking recipes for 0.5 to $4 \mathrm{GHz}$

\begin{tabular}{lccc}
\hline Ingredient & Bone Cortical & Blood & Muscle \\
\hline Flour $(\mathrm{g})$ & 40 & - & - \\
Sugar $(\mathrm{g})$ & 3.6 & 45.5 & - \\
Salt $(\mathrm{g})$ & - & 0.5 & - \\
Deionized water $(\mathrm{ml})$ & 5.5 & 82.5 & 100 \\
Gelatine $(\mathrm{g})$ & - & - & 5 \\
Detergent (ml) & - & - & 15.5 \\
Oil (ml) & 15.2 & - & 15.5 \\
Food colouring (ml) & - & 0.5 & - \\
\hline
\end{tabular}




\subsection{Measurement setup using a three material phantom}

At first, to ensure correct implantation of the monopoles in agreement with the $5 \mathrm{~mm}$ gap of free space between the phantoms and the groundplanes according to the test-bed specifications of Section 2, the PLA insulators of the monopoles were first implanted into the middle of the bone layer. The insulators were separated by a gap of $20 \mathrm{~mm}$ from each other (centre to centre); then, the bone emulating tissue was placed inside the muscle phantom and its exposed top surface was carefully covered until all of the air cavities were filled with the muscle phantom (see Figure 3). The monopoles were then inserted into the insulating sleeves. The diameters of the bone tissues for all the phantoms presented in Sections 2.3.1 to 2.3.3, were given according to the average diameter of their equivalent bones provided by the Voxel model of CST Microwave Studio [33].

\subsubsection{Forearm-radius}

The length of each monopole for the measurement setup of the radius bone was chosen to be $40 \mathrm{~mm}$. The dimensions of the groundplanes were $60 \times 56 \mathrm{~mm}$. The groundplanes were attached to the parts of the monopoles in free space at the surface of the phantom (see Figure 4). The 40mm gap between the groundplanes was used as an entry point for the injection of the blood emulating liquid via a sterilized syringe of $1 \mathrm{~mm}$ diameter. Although the envisioned $5 \mathrm{~mm}$ air gap between the phantom and the groundplanes was followed precisely, the groundplanes were also insulated using plastic sticky tape, thus eliminating the possibility of contact with the highly conductive muscle layer.

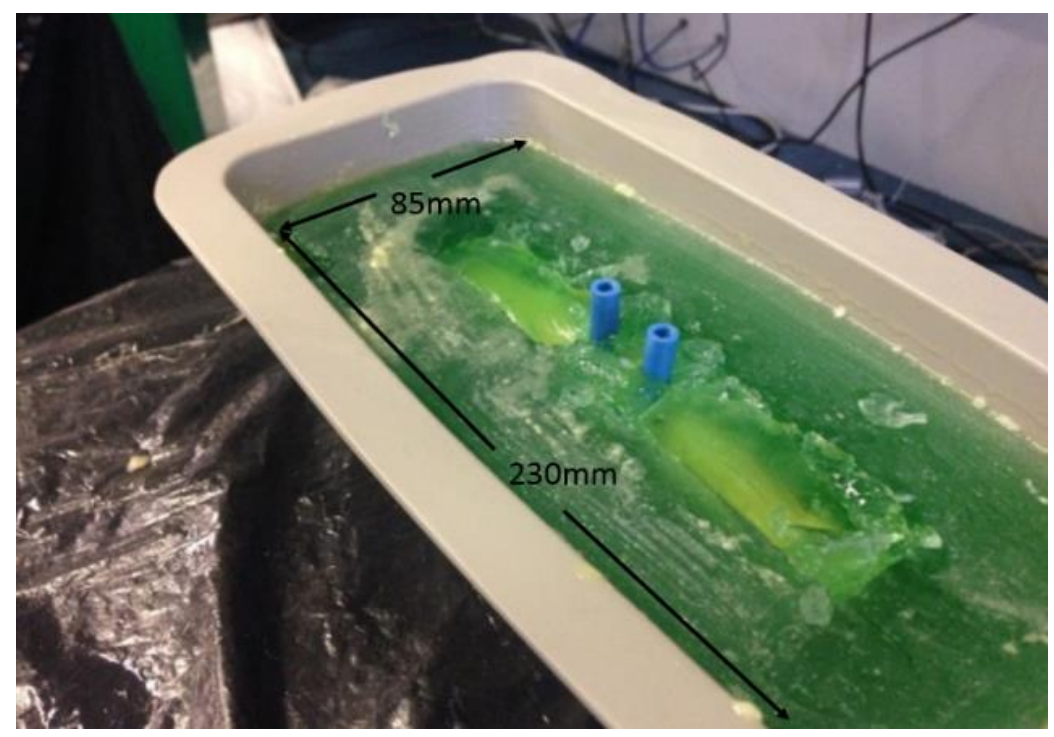

Figure 3: Radius phantom with implanted insulators. 
The diameter of the bone layer was approximately $15 \mathrm{~mm}$ and its length was $160 \mathrm{~mm}$. The dimensions of the muscle layer were $230 \times 85 \times 50 \mathrm{~mm}$ and a maximum of $30 \mathrm{ml}$ of blood emulating liquid was injected between the monopoles for the final measurement.

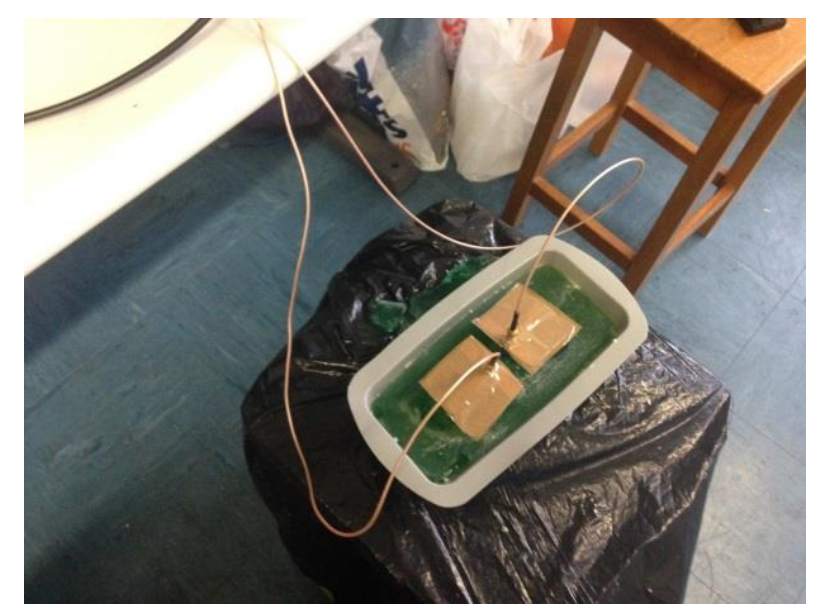

Figure 4: Monopoles implanted into the radius phantom

\subsubsection{Finger - phalange}

For the phalange phantom (see Figure 5), the bone layer had $7.5 \mathrm{~mm}$ diameter approximately and 60mm length. The muscle layer's dimensions were $90 \times 65 \times 40 \mathrm{~mm}$. The monopoles were $25 \mathrm{~mm}$ long and the dimensions of the groundplanes were $60 \times 40 \mathrm{~mm}$. Due to the smaller size of the phalange bone and its equivalent phantom, the maximum amount of blood emulating liquid that was injected between the monopoles was $10 \mathrm{ml}$ in five injections of $2 \mathrm{ml}$.

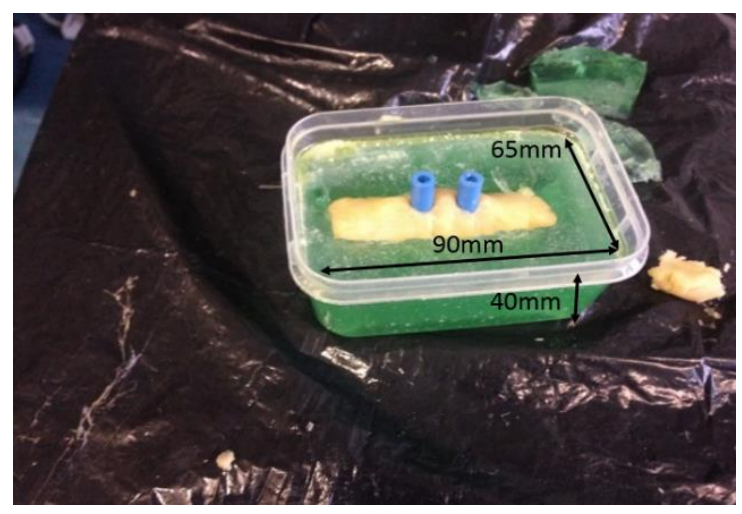

Figure 5: Bone layer and PLA insulators 


\subsubsection{Leg-tibia}

For the measurements of the tibia phantom, the diameter of the bone layer was approximately $20 \mathrm{~mm}$ and the length was $160 \mathrm{~mm}$ (see Figure 6). The dimensions of the muscle layer were $230 \times 85 \times 50 \mathrm{~mm}$. The length of the monopoles was $25 \mathrm{~mm}$ and the dimensions of the groundplanes were $60 \times 40 \mathrm{~mm}$.

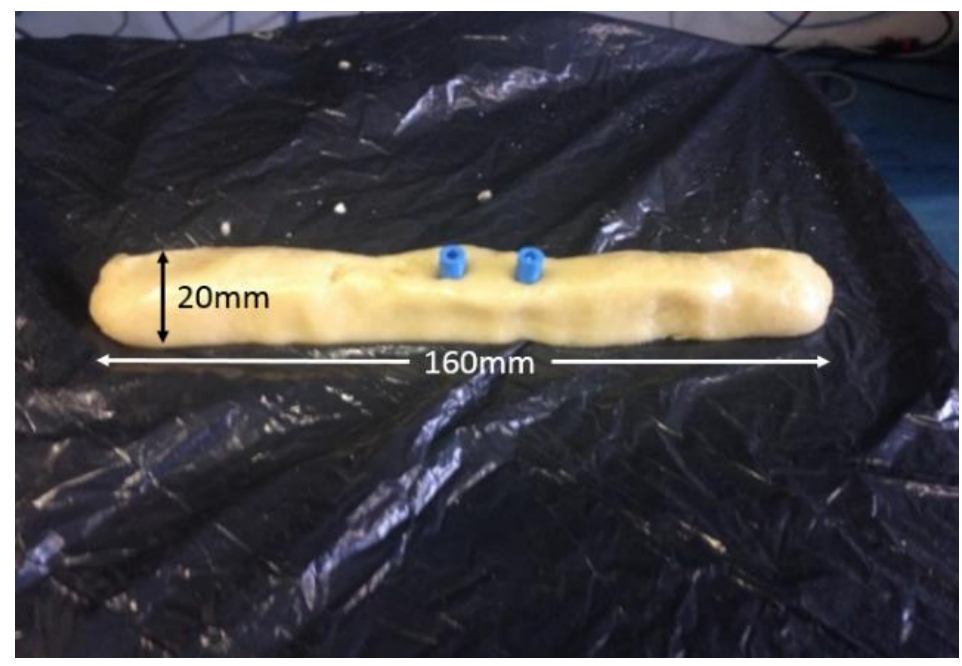

Figure 6: Bone layer and PLA insulators

For each phantom setup presented above, the $S_{21}$ of the monopoles was measured using an Anritsu MS46524A vector network analyzer that was calibrated over the 1 to $4 \mathrm{GHz}$ frequency range. The first measurement was conducted without injecting any blood emulating liquid into the phantom to emulate the fully healed condition of the bone. Each of the next measurements were taken for every $2 \mathrm{ml}$ of blood emulating liquid that was injected into each phantom into the bone layer in the mid distance between the monopoles until the maximum amount of millilitres was reached.

\subsection{Ex-vivo lamb joint measurement}

For the ex-vivo measurements, a lamb femur joint was used. Two $4 \mathrm{~mm}$ diameter holes were drilled in the top surface of the bone for the monopole insulators to be implanted. The $25 \mathrm{~mm}$ length monopoles that were used in the phalange and tibia phantoms were selected for implantation due to the $20 \mathrm{~mm}$ diameter of the bone joint. The distance between the holes was measured as $20 \mathrm{~mm}$ as shown in Figure 7. The first $S_{21}$ measurement was taken without fracturing the bone. For the $S_{21}$ measurements that followed, a $4 \mathrm{~mm}$ diameter hole was drilled in the mid distance between the monopoles (position 1 in Figure 7) and $2 \mathrm{ml}$ of blood emulating liquid was added for each step, up to the amount of $10 \mathrm{ml}$. Then, a second $4 \mathrm{~mm}$ diameter hole was drilled just to the right of the first hole (position 2 in Figure 7). The liquid injection process continued in $2 \mathrm{ml}$ steps with an $S_{21}$ measurement at each step 
until a total of $20 \mathrm{ml}$ had been injected. Finally, a third $4 \mathrm{~mm}$ hole was drilled just to the left of the first hole (position 3 in Figure 7) and the injection and $S_{21}$ measurement process continued in steps of $2 \mathrm{ml}$ until a total of $30 \mathrm{ml}$ of liquid was added to the fracture.

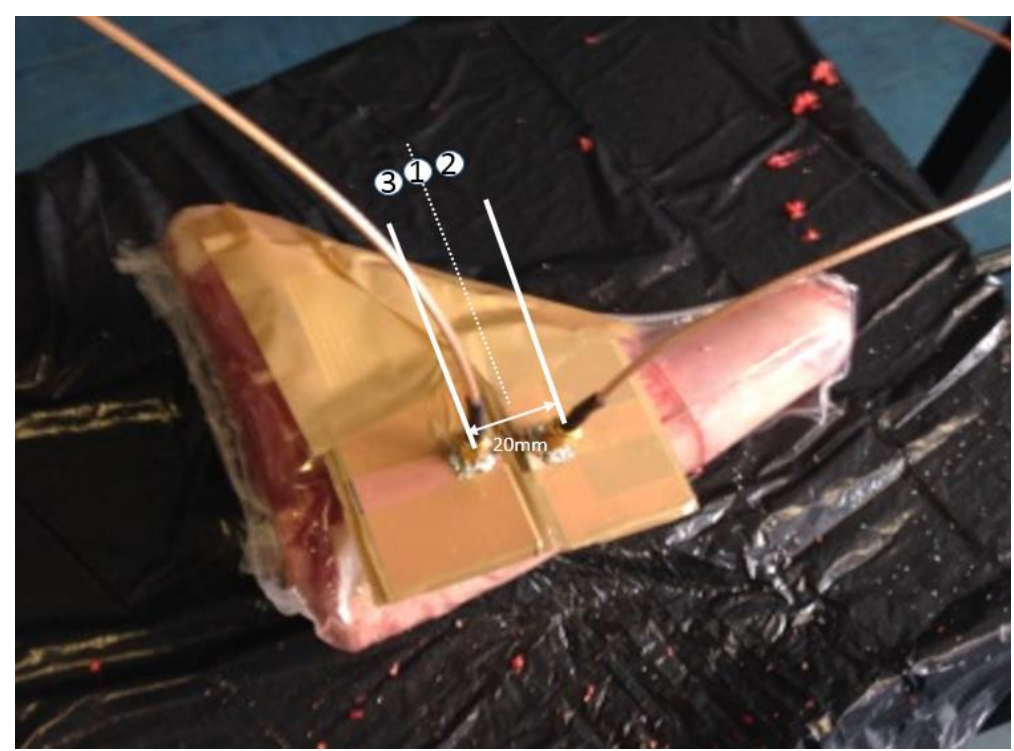

Figure 7: Drilling sequence of the holes into the lamb joint

\section{Results and discussion}

The phantoms of radius, phalange and tibia bones were developed for the assessment of an antenna system as a tool for fracture monitoring. Dielectric measurements were also taken in the centre of the fracture for the same amounts of injected blood emulating liquid using the coaxial probe of Section 2.3 in all three phantoms as a measurement validation technique. The volume ratio of bone versus blood emulating liquid inside the fractured area was calculated based on the measured relative permittivity of the investigated area compared to the relative permittivity of the bone and the blood tissues (see Figure 8). Radius fracture consisted of $24.1 \%$ blood at $10 \mathrm{ml}$, $63.7 \%$ blood at $20 \mathrm{ml}$ and $97.1 \%$ blood at $30 \mathrm{ml}$. phalange fracture consisted of $38.2 \%$ blood at $6 \mathrm{ml}, 64.7 \%$ blood at $8 \mathrm{ml}$ and $90.0 \%$ blood at $10 \mathrm{ml}$. Tibia fracture consisted of $36.9 \%$ blood at $10 \mathrm{ml}, 51.7 \%$ blood at $20 \mathrm{ml}$ and $91.5 \%$ blood at $30 \mathrm{ml}$. According to measurements conducted in [16], the measured relative permittivity of the Trabecular bone callus that signals bone unification varies between 35 and 45 at $1 \mathrm{GHz}$. That range of values show the closest agreement with the $8 \mathrm{ml}$ of injected blood emulating liquid measurement for the phalange phantom and with the $20 \mathrm{ml}$ for the tibia and radius phantoms (see Figure 8). 


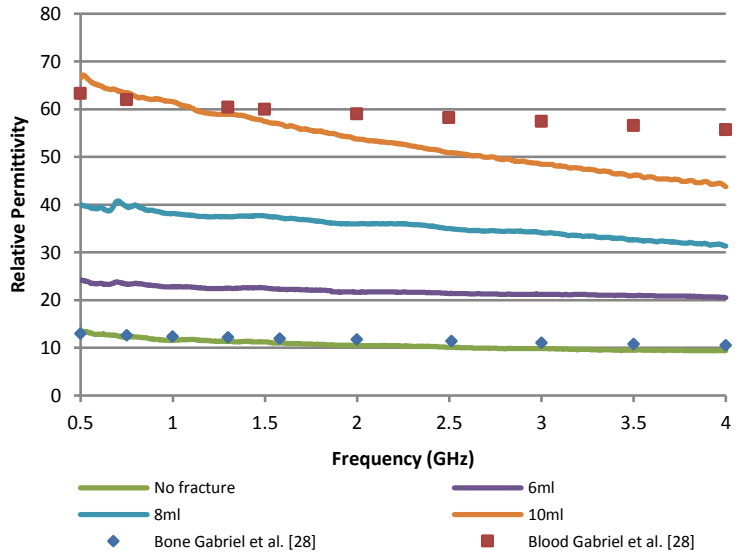

(a)

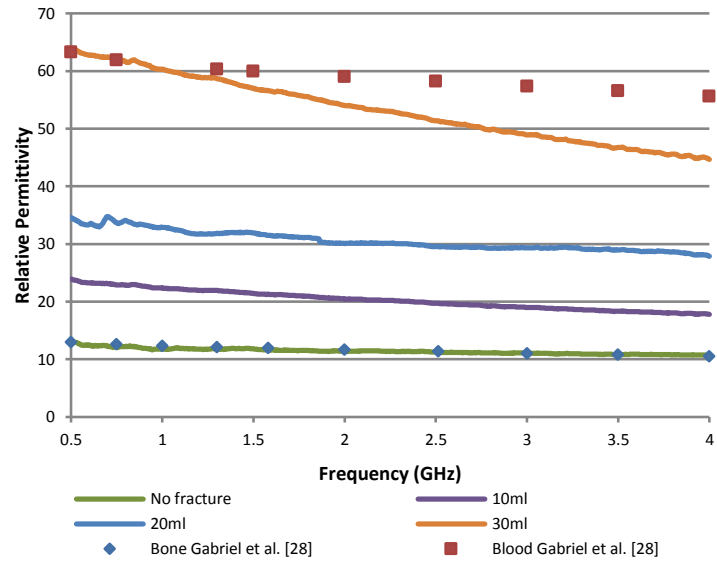

(b)

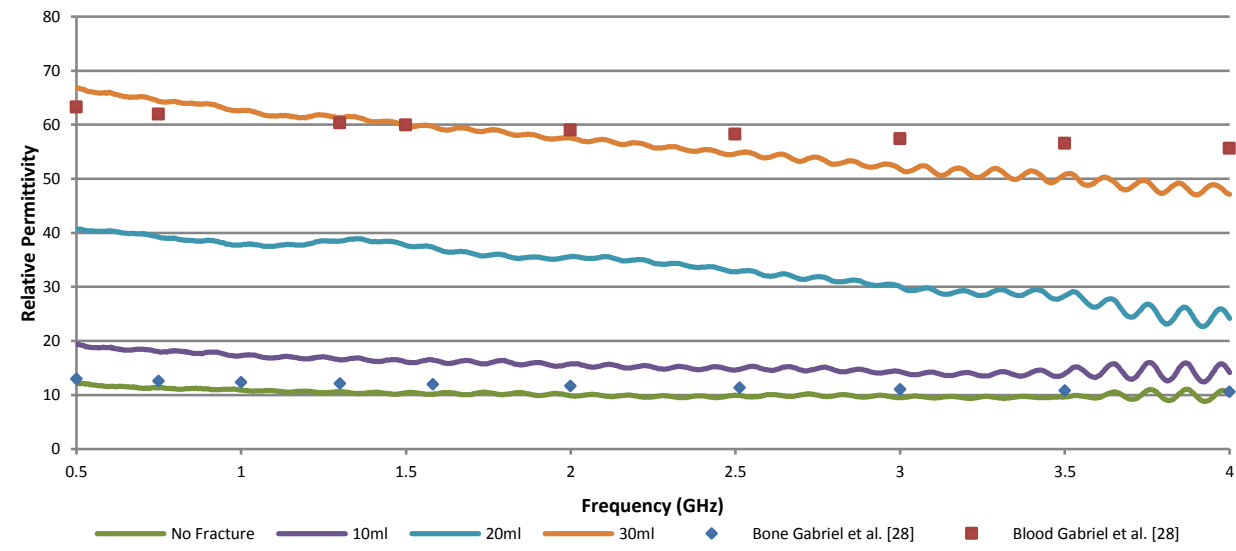

(c)

Figure 8: Coaxial probe dielectric measurements in the middle of the bone fracture emulating region as different amounts of blood were added in: a) Phalange, b) tibia, c) radius phantoms

The presented measurement results in Figures 9, 10 and 11 show that by gradually increasing the volume of blood emulating liquid between the monopoles, the magnitude of the $S_{21}$ attenuated over the investigated frequency spectrum. Lamb joint measurements in Figure 12 confirm the $S_{21}$ magnitude attenuation in ex-vivo tissues. Several resonances created in the measurements compared to the smoother plots of simulations are due to the energy trapped in the cavities created by the complex geometry of the dispersion of the blood emulating liquid inside the phantoms (see Figures 9 to 11, and further analysis at the end of this section). 


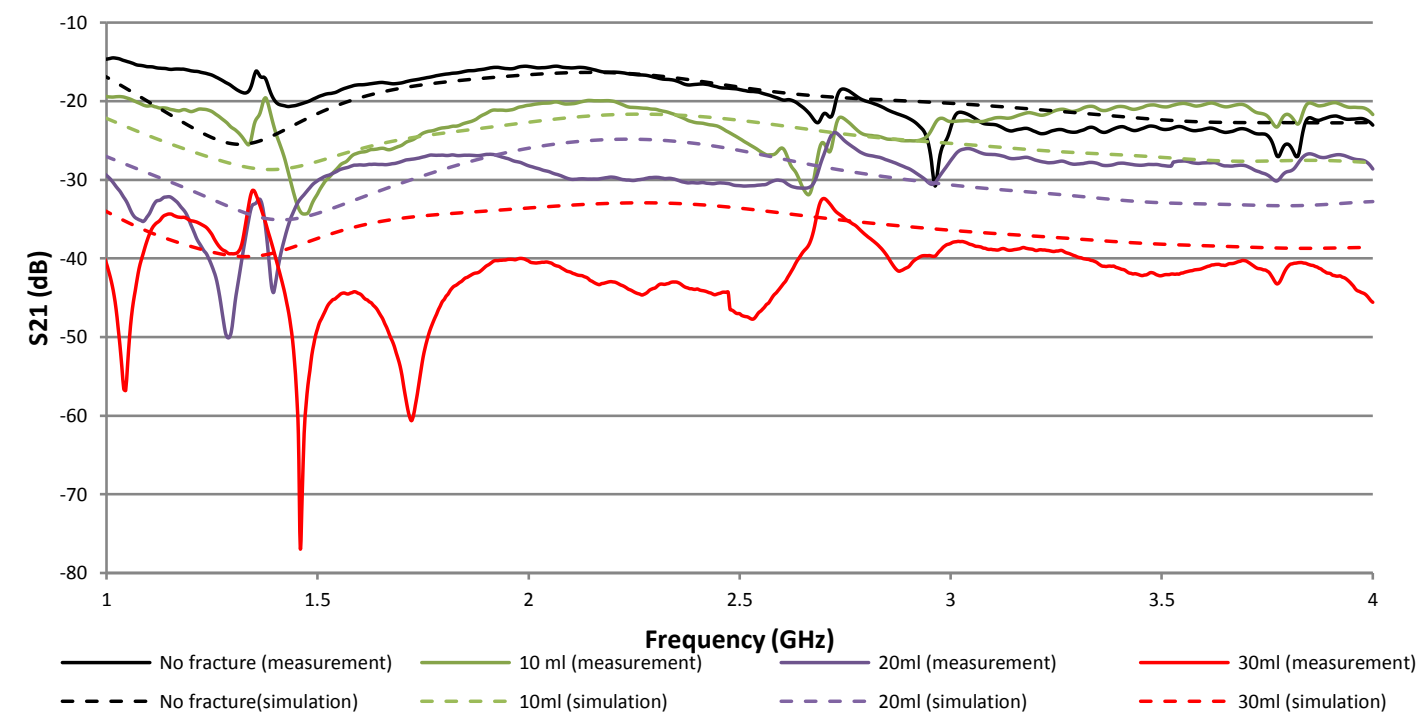

Figure 9: The $\boldsymbol{S}_{\mathbf{2 1}}$ measurement and simulation results for the radius phantom with different quantities of blood representing the fracture healing.

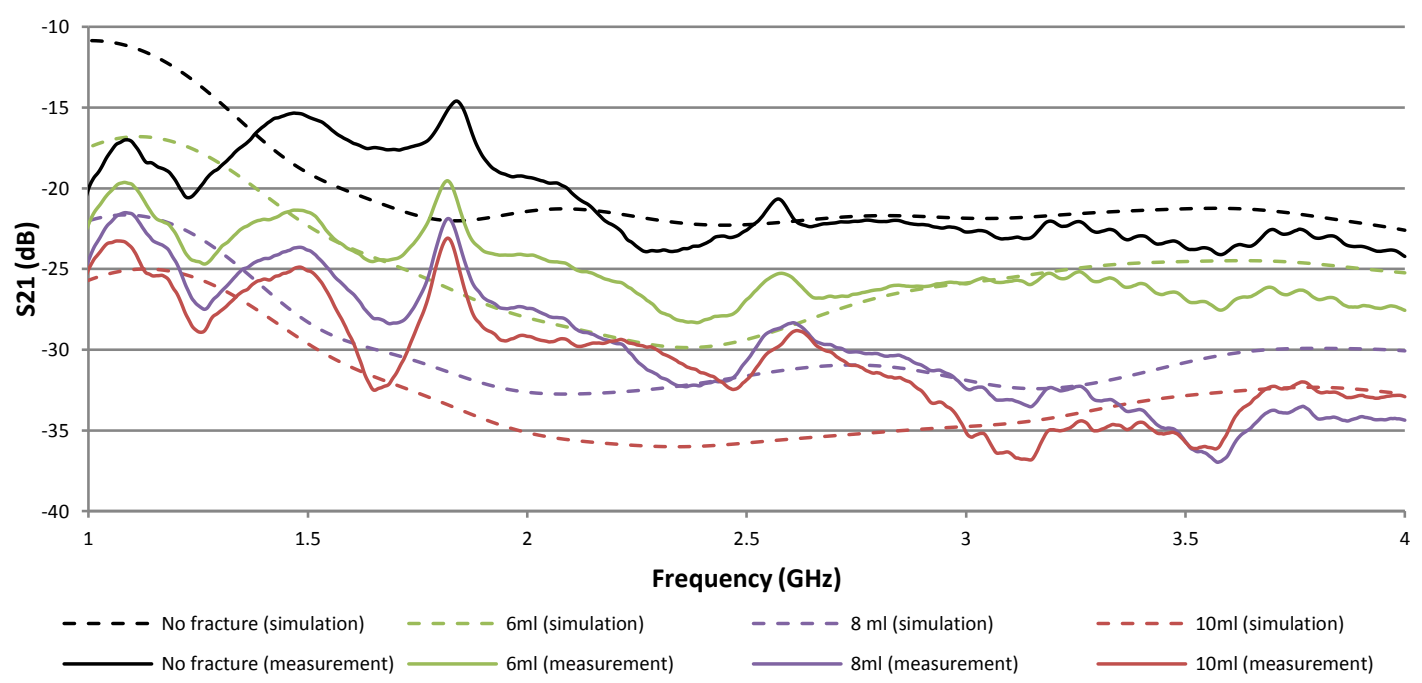

Figure 10: The $\boldsymbol{S}_{\mathbf{2 1}}$ measurement and simulation results for the phalange phantom with different quantities of blood representing the fracture healing 


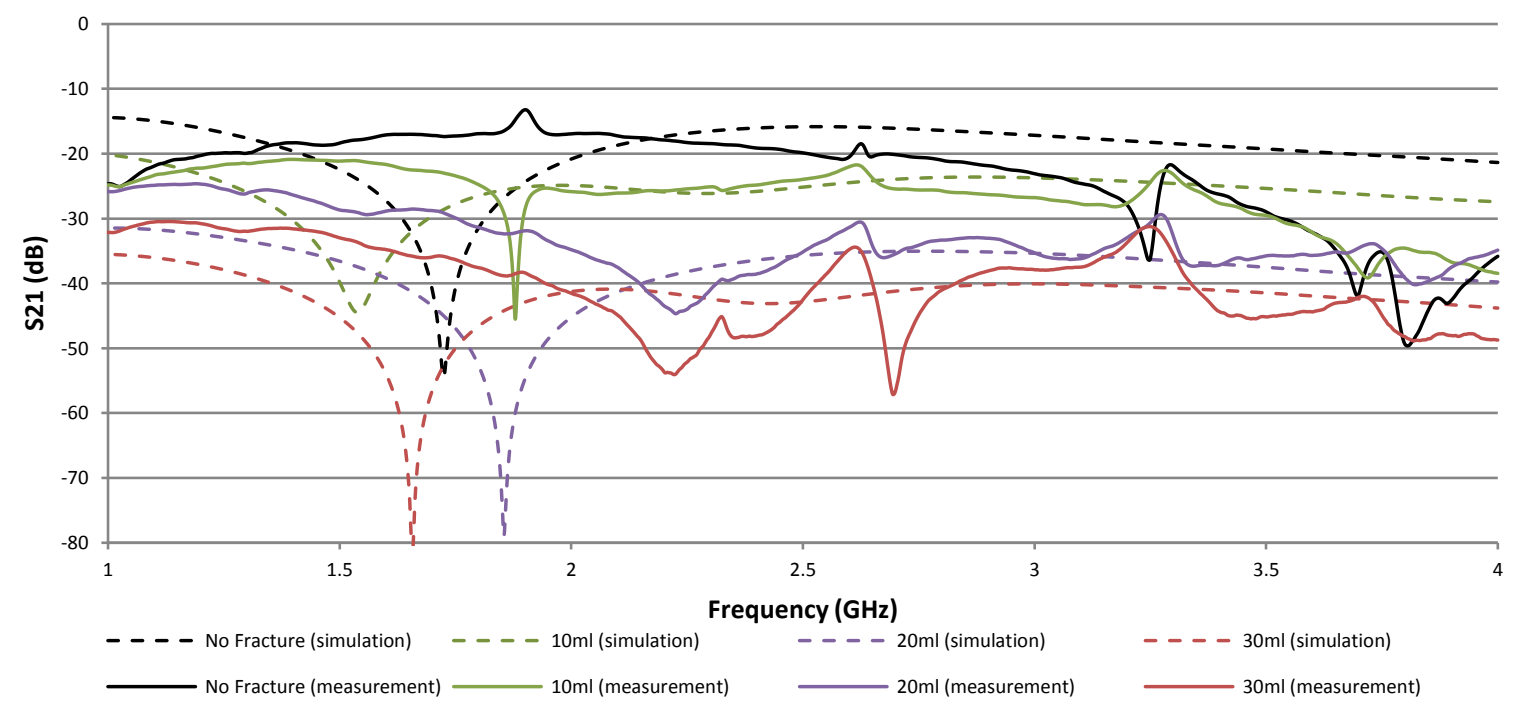

Figure 11: The $\boldsymbol{S}_{\mathbf{2 1}}$ measurement and simulation results for the tibia phantom with different quantities of blood emulating liquid representing the hematoma - fracture reduction.

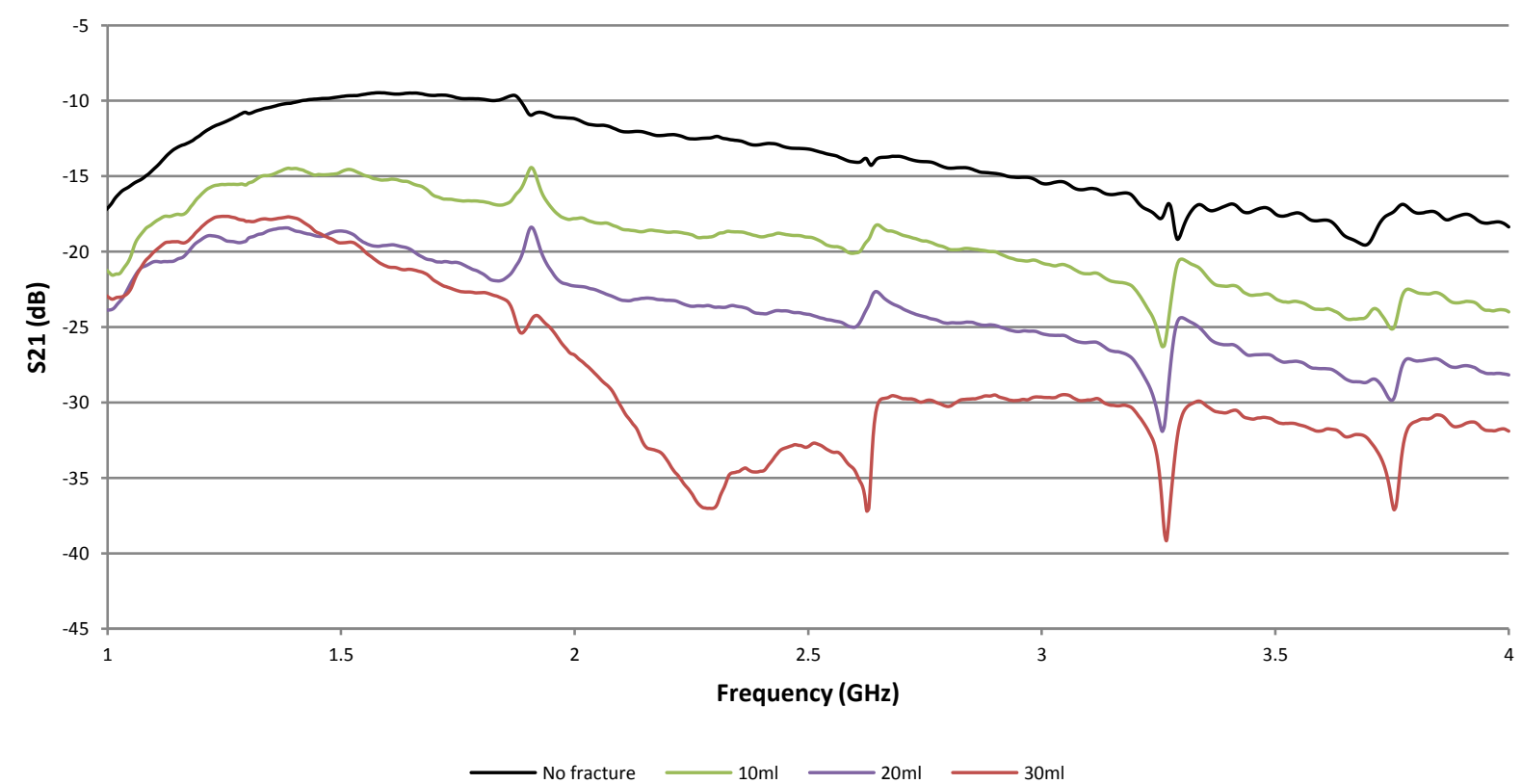

Figure 12: The $\boldsymbol{S}_{\mathbf{2 1}}$ measurement results for the lamb joint with different quantities of blood emulating liquid representing the hematoma - fracture reduction. 
The attenuation of the $S_{21}$ due to the high relative permittivity and conductivity of blood can be additionally observed in Figure 13 where the electric fields of the monopoles are depicted inside a simulated multi-material geometrical phantom that features the exact dimensions of the fabricated tibia bone phantom of Section 2.3.3 with and without the blood cylinders representing the bone fracture.

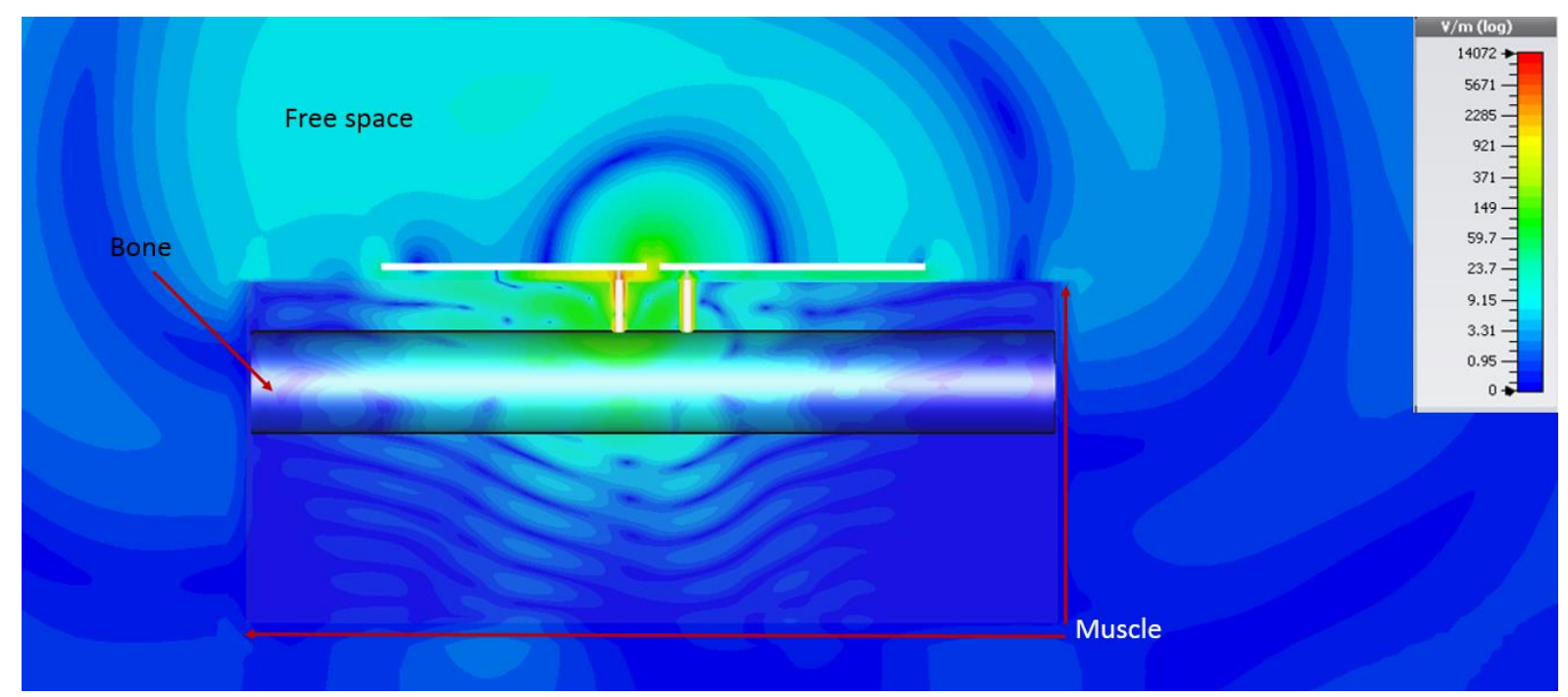

(a)

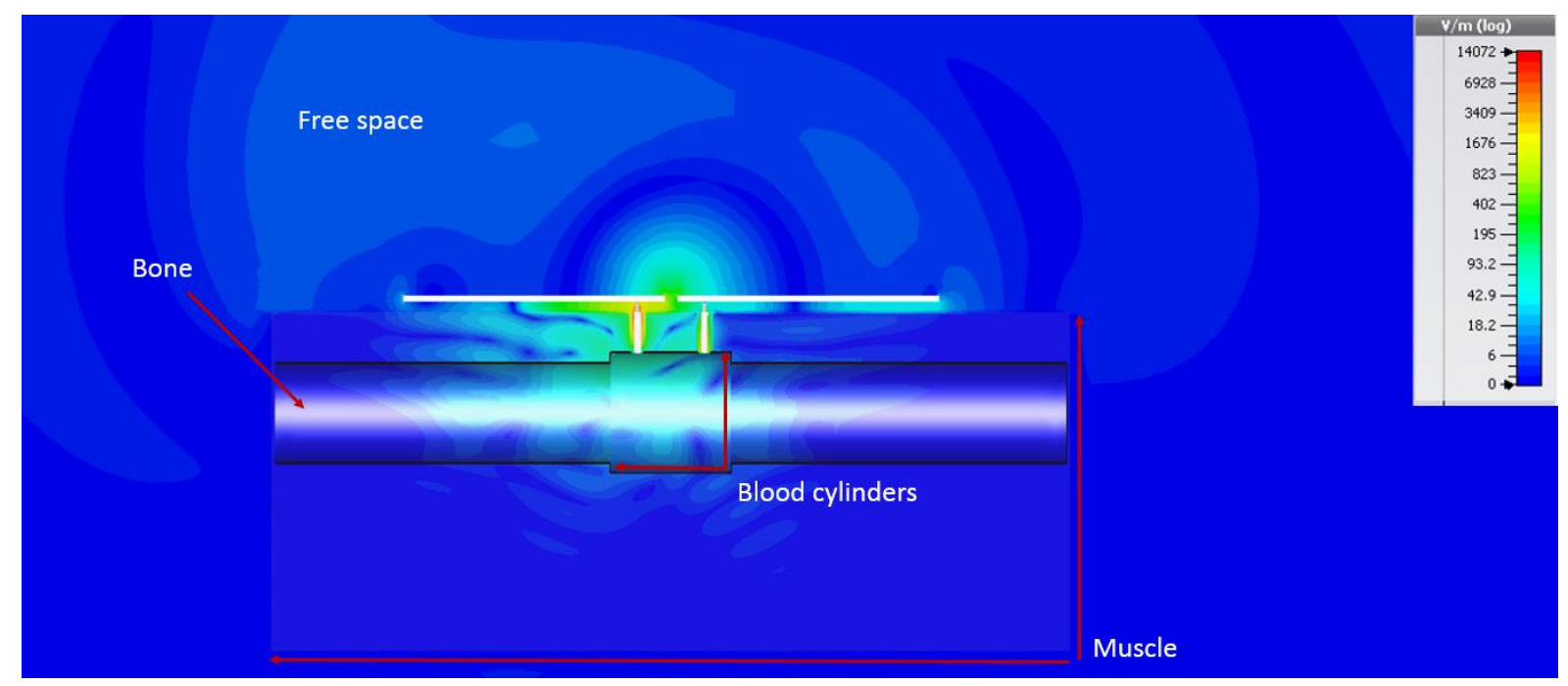

(b)

Figure 13: The electric field of the monopoles inside a simulated geometric tibia phantom with the same dimensions as the fabricated phantom a) with a blood cylinder, b) without a blood cylinder

The comparison between Figure 13a and Figure 13b shows that the magnitude of the electric field is greatly reduced inside the muscle and in free space when the blood cylinders are introduced by replacing the bone in the structure. The electric field of the monopoles in the far-field region is described by [40]: $\vec{E}(\vec{r})=\overrightarrow{E_{0}} e^{-j \vec{k} \vec{r}}$, (the harmonic time-dependence $e^{j \omega t}$ is assumed), where $E_{0}$ is the magnitude of the electric field on the surface of the monopole. The exponential $e^{-j k r}$ of the far field equation describes the attenuation of the propagating electric field inside a medium of wavenumber $k$ at a distance $r$. The wavenumber $k$ depends on the relative permittivity, 
$\varepsilon_{r}$, and conductivity, $\sigma$, of each simulated tissue and is given by: $k=|\vec{k}|=\omega \sqrt{\mu_{0} \mu_{r} \varepsilon_{0}\left(\varepsilon_{r}-j \frac{\sigma}{\omega \varepsilon_{0}}\right)}$, where $\omega$ is the angular frequency and $\mu_{0}, \varepsilon_{0}$ are the permeability and permittivity of vacuum respectively. The relative permittivity and conductivity of blood are higher than that of bone over the frequency range 1 to $4 \mathrm{GHz}[17,18$, 38], i.e. $\varepsilon_{\text {rblood }}=61, \sigma_{\text {blood }}=1.58$ and $\varepsilon_{\text {rbone }}=12, \sigma_{\text {bone }}=0.15$ at $1 \mathrm{GHz}$. Therefore, the attenuation of the electric field is greater when the blood cylinder or blood emulating liquid is introduced inside the phantom for simulations and measurements respectively, having as a result a worsening in the $S_{21}$ magnitude as the volume of the blood increases (see Figures $13 \mathrm{a} \& 13 \mathrm{~b}$ ). The $S_{21}$ attenuation in the simulations was broadly validated in measurements as the amount of blood increased in volume inside the bone, one difference being the additional resonances in the $S_{21}$ seen in the measurements. In the ex-vivo measurements, it is not possible to create perfect geometric cylindrical shapes for the blood-emulating liquid. Creating a fracture with three circular holes only adds to the complexity of the bone's geometry. Furthermore, small bone fragments combined with the blood-emulating liquid and bone marrow is unlikely to perfectly mimic the homogenous blood properties of the simulation. As a result, the mixture inside the bone is likely to pose multiple different dielectric layers to the propagating wave. These are the likely causes of standing waves and resonances in the $S_{21}$ in the measurements.

Since the relative permittivity and conductivity of blood is significantly higher than that of bone across much of the microwave frequency range, the pattern of change of $S_{21}$ is likely be observed for much of this frequency range. Therefore, the monopole lengths could easily be adapted to fit the diameter and depth of any human bone likely to have external fixators. However, it is important to bear in mind microwave attenuation increases dramatically with increasing frequency. So in order to obtain a measureable $S_{21}$ with low noise, it would be advisable to conduct measurements in the 1 to $4 \mathrm{GHz}$ frequency range.

Finally, the opposite process where the magnitude of the $S_{21}$ increases after a bone fracture due to the reduction of blood, can provide an indication of the healing in the fractured area during the first four weeks after the trauma.

\section{Conclusions}

A technique for monitoring the healing of severe bone fractures, using a pair of radio frequency monopoles implanted in the bone was investigated in this work. The magnitude of change of the $S_{21}$ measurements of two implanted monopoles in the radius, phalange and tibia phantoms has shown to provide a good indication regarding the healing progress of different types of bone fractures that doctors use external metal plates for their treatment. Our preliminary results have shown that, as the injected blood emulating liquid's volume increased inside the fracture, the transmission between the two monopoles is severely attenuated. This was due to the high relative permittivity and conductivity of the liquid. The results were validated in an ex-vivo measurement of a bone fracture in a lamb joint. The proposed method could provide a fracture monitoring technique during the first four weeks after the trauma. In a clinical context, no two fractures would ever be identical. The $\mathrm{S}_{21}$ that corresponds to a unified fracture $15 \mathrm{~cm}$ down from the head of the femur in an elderly male is unlikely to be the same as a fracture $25 \mathrm{~cm}$ down from the head of a femur in a young female. When the size of the fracture, the distance between the monopoles and their lengths are taken into account, there will be considerable variation in $S_{21}$ between patients with consolidated fractures. Therefore, from this study at least, it would not be appropriate to 
equate an absolute value of $S_{21}$ to consolidated fractures in general. Instead, the focus should be on the rate of change of $S_{21}$ magnitude over time. If a fracture is healing in the normal way, the blood haematoma will reduce over time and there will be an associated continual improvement in the $S_{21}$. If the healing time can be estimated by the clinicians, then one would expect the rate of change of $S_{21}$ to slow as that period comes to an end. If however, the rate of change of $S_{21}$ drops well in advance of the estimated healing time, this system would indicate healing has stalled and would warrant further investigation by the clinicians.

Further work is needed to develop an analytical model for the near-field coupling between two monopoles of arbitrary separation distance inside bone. This can be combined with computer simulation studies using different anatomical human models looking at $S_{21}$ magnitudes in non-fractured bones that are likely to use external fixators. If successful, this could make it possible for clinicians to predict a $S_{21}$ range for a consolidated fracture in any bone and for any monopole separation distance. Further work is also required on how to maintain mechanical stability of the fixator when steps are taken to electrically isolate the monopoles form their ground planes. All theories and methodologies proposed here will have to be tested in animal studies.

\section{Acknowledgments}

The authors would like to thank Dr. Athanasios Goulas of the Wolfson School of Mechanical, Electrical and Manufacturing Engineering at Loughborough University for the support in the design of the 3D printed monopole insulators. The authors would also like to acknowledge the partial financial support of the Engineering and Physical Sciences Research Council for this work via the UNIFY[41] and UNIFY+[42] Networks.

\section{References}

[1] J. Kim and Y. Rahmat-Samii, "Implanted antennas inside a human body: Simulations, designs, and characterizations," IEEE Transactions on Microwave Theory and Techniques, vol. 52, no. 8, pp. 1934$1943,2004$.

[2] P. Soontornpipit, C. M. Furse, and Y. C. Chung, "Design of implantable microstrip antenna for communication with medical implants," IEEE Transactions on Microwave Theory and Techniques, vol. 52, no. 8, pp. 1944-1951, 2004.

[3] T. Karacolak, A. Z. Hood, and E. Topsakal, "Design of a dual-band implantable antenna and development of skin mimicking gels for continuous glucose monitoring," IEEE Transactions on Microwave Theory and Techniques, vol. 56, no. 4, pp. 1001-1008, 2008.

[4] W. Xia, K. Saito, M. Takahashi, and K. Ito, "Performances of an implanted cavity slot antenna embedded in the human arm," IEEE Transactions on Antennas and Propagation, vol. 57, no. 4, pp. 894-899, 2009.

[5] R. Warty, M. R. Tofighi, U. Kawoos, and A. Rosen, "Characterization of implantable antennas for intracranial pressure monitoring: Reflection by and transmission through a scalp phantom," IEEE 
Transactions on Microwave Theory and Techniques, vol. 56, no. 10, pp. 2366-2376, 2008.

[6] S. Soora, K. Gosalia, M. S. Humayun, and G. Lazzi, "A comparison of two and three dimensional dipole antennas for an implantable retinal prosthesis," IEEE Transactions on Antennas and Propagation, vol. 56, no. 3, pp. 622-629, 2008.

[7] T. Karacolak, R. Cooper, and E. Topsakal, "Electrical properties of rat skin and design of implantable antennas for medical wireless telemetry," IEEE Transactions on Antennas and Propagation, vol. 57, no. 9, pp. 2806-2812, 2009.

[8] J. Abadia, F. Merli, J. F. Zurcher, J. R. Mosig, and A. K. Skrivervik, “3D-Spiral small antenna design and realization for biomedical telemetry in the MICS band," Radioengineering, vol. 18, no. 4, pp. 359$367,2009$.

[9] E. Y. Chow, C. L. Yang, A. Chlebowski, S. Moon, W. J. Chappell, and P. P. Irazoqui, "Implantable wireless telemetry boards for in Vivo transocular transmission," IEEE Transactions on Microwave Theory and Techniques, vol. 56, no. 12, pp. 3200-3208, 2008.

[10] Z. N. Chen, G. C. Liu, and T. S. P. See, "Transmission of RF signals between MICS loop antennas in free space and implanted in the human head," IEEE Transactions on Antennas and Propagation, vol. 57, no. 6, pp. 1850-1854, 2009.

[11] K. Gosalia, M. S. Humayun, and G. Lazzi, "Impedance matching and implementation of planar spacefilling dipoles as intraocular implanted antennas in a retinal prosthesis," IEEE Transactions on Antennas and Propagation, vol. 53, no. 8, pp. 2365-2373, 2005.

[12] P. M. Izdebski, H. Rajagopalan, and Y. Rahmat-Samii, "Conformal ingestible capsule antenna: A novel chandelier meandered design," IEEE Transactions on Antennas and Propagation, vol. 57, no. 4, pp. 900-909, 2009.

[13] M. W. A. Khan, T. Bjorninen, L. Sydanheimo, and L. Ukkonen, "Characterization of Two-Turns External Loop Antenna With Magnetic Core for Efficient Wireless Powering of Cortical Implants,” IEEE Antennas and Wireless Propagation Letters, vol. 15, pp. 1410-1413, 2016.

[14] V. Perlepe, P. Omoumi, F. Lecouvet, and B. Vande Berg, "A radiographic and CT scoring system for quantification of the healing process in delayed-unions of long bone fractures: a feasibility study," in ECR (European Society of Radiology), 2013, pp. 1-13.

[15] T. A. Einhorn and L. C. Gerstenfeld, "Fracture healing: mechanisms and interventions," Nature Reviews Rheumatology, vol. 11, no. 1, pp. 45-54, Jan. 2015.

[16] R. M. Irastorza, S. Valente, F. Vericat, and E. Blangino, "Dielectric properties in fresh trabecular bone tissue from $1 \mathrm{MHz}$ to $1000 \mathrm{MHz}$ : A fast and non destructive quality evaluation technique," Materials Science Forum, vol. 638-642, pp. 730-735, 2010. 
[17] C. Spross, R. Zeledon, V. Zdravkovic, and B. Jost, "How bone quality may influence intraoperative and early postoperative problems after angular stable open reduction - internal fixation of proximal humeral fractures," Journal of Shoulder and Elbow Surgery, pp. 1-7, 2017.

[18] S. Symeonidis, C. Torres-Sanchez, C. Panagamuwa, and W. G. Whittow, “An Implanted Antenna System for the Monitoring of Bioresorbability of a Biocompatible Scaffold Embedded into a Bone Fracture," in MOBIHEALTH'15 Proceedings of the 5th EAI International Conference on Wireless Mobile Communication and Healthcare, 2015, pp. 1-4.

[19] S. Symeonidis, W. G. Whittow, C. J. Panagamuwa, and M. Zecca, “An Implanted Antenna System for the Monitoring of the Healing of Bone Fractures," in 2015 Loughborough Antennas \& Propagation Conference (LAPC), 2015, pp. 1-4.

[20] P. J. Harwood, J. B. Newman, and A. L. R. Michael, “(ii) An update on fracture healing and non-union," Orthopaedics and Trauma, vol. 24, no. 1, pp. 9-23, 2010.

[21] K. D. Hankenson, G. Zimmerman, and R. Marcucio, "Biological perspectives of delayed fracture healing," Injury, vol. 45, pp. S8-S15, 2014.

[22] P. Elsissy, Y. T. Akpolat, A. Chien, and W. K. Cheng, "MRI evaluation of the knee with nonferromagnetic external fixators: cadaveric knee model," European Journal of Orthopaedic Surgery and Traumatology, vol. 25, no. 5, pp. 933-939, 2015.

[23] M. Khan, S. P. S. M. A. Sirdeshmukh, and K. Javed, "Evaluation of bone fracture in animal model using bio-electrical impedance analysis," Perspectives in Science, vol. 8, pp. 567-569, 2016.

[24] K. Gupta, P. Gupta, G. Singh, S. Kumar, R. Singh, and R. Srivastava, "Change in electrical properties of bone as diagnostic tool for measurement of fracture healing," Hard Tissue, vol. 2, no. 1, pp. 1-8, 2013.

[25] T. Yoshida, W.-C. Kim, Y. Oka, N. Yamada, and T. Kubo, “Assessment of distraction callus in rabbits by monitoring of the electrical impedance of bone.," Acta orthopaedica, vol. 81, no. 5, pp. 628-633, 2010.

[26] R. S. Lakes, R. A. Harper, and J. L. Katz, "Dielectric relaxation in cortical bone," Journal of Applied Physics, vol. 48, no. 2, pp. 808-811, 1977.

[27] C. Goldstein, S. Sprague, and B. a Petrisor, "Electrical stimulation for fracture healing: current evidence," Journal of Orthopaedic Trauma, vol. 24, no. 3, pp. 62-65, 2010.

[28] R. Pethig and D. B. Kell, "The passive electrical properties of biological systems: their significance in physiology, biophysics and biotechnology.," Physics in medicine and biology, vol. 32, no. 8, pp. 933970, 1987.

[29] T. Hirashima, W.-C. Kim, W.-C. Kim, K. Kawamoto, T. Yoshida, and T. Kubo, "Evaluating bone union of distal radius fractures by measuring impedance values," Orthopedics, vol. 32, no. 1, pp. 1-31, 2009. 
[30] R. Wade and J. Richardson, “Outcome in fracture healing: A review,” Injury, vol. 32, no. 2, pp. 109114,2001

[31] C. Gabriel, "Compilation of the Dielectric Properties of Body Tissues at RF and Microwave Frequencies.," Environmental Health, pp. 1-21, 1996.

[32] “Reference Chart of Screws, Drill Bits, Taps and Guide Wires.” [Online]. Available: http://www.cambridgeorthopaedics.com/. [Accessed: 20-Apr-2018].

[33] “CST Homepage.” [Online]. Available: https://www.cst.com/. [Accessed: 31-Jul-2017].

[34] R. A. D. Carano and E. H. Filvaroff, “Angiogenesis and bone repair,” Drug Discovery Today, vol. 8, no. 21, pp. 980-989, 2003.

[35] S. Symeonidis, W. G. Whittow, and C. Panagamuwa, "Design and characterization of a three material anatomical bone phantom for implanted antenna applications," in Loughborough Antennas \& Propagation Conference (LAPC), 2017, pp. 4-8.

[36] C. J. Panagamuwa, I. Howells, and W. G. Whittow, "Conductivity and permittivity measurements of children and adult's hands covering mobile communications frequency bands," Progress In Electromagnetics Research Symposium (PIERS), pp. 1-5, 2013.

[37] J. H. Jung, S. W. Kim, Y. S. Kim, and S. Y. Kim, "Electromagnetic propagation from the intestineingested source in the human body model," IEEE Transactions on Antennas and Propagation, vol. 58, no. 5, pp. 1683-1688, 2010.

[38] D. M. Hagl, D. Popovic, S. C. Hagness, J. H. Booske, and M. Okoniewski, "Sensing volume of openended coaxial probes for dielectric characterization of breast tissue at microwave frequencies," IEEE Transactions on Microwave Theory and Techniques, vol. 51, no. 4 I, pp. 1194-1206, 2003.

[39] S. Symeonidis, W. G. Whittow, and C. J. Panagamuwa, "Characterisation of an Antenna System Implanted Into a Limb Phantom for Monitoring of Bone Fracture Healing," in Loughborough Antennas \& Propagation Conference (LAPC), 2016.

[40] C. A. Balanis, Modern Antenna Handbook. New York, NY, USA: Wiley-Interscience, 2008.

[41] EPSRC, “EPSRC - NIHR Healthcare Technology Co-operative (HTC) Partnership Award - Unify: A network focused on the treatment of fracture non-unions." [Online]. Available: http://gow.epsrc.ac.uk/NGBOViewGrant.aspx?GrantRef=EP/M000230/1. [Accessed: 02-Aug-2017].

[42] EPSRC, “EPSRC-NIHR HTC Partnership Award 'Plus': UNIFY Plus.” [Online]. Available: http://gow.epsrc.ac.uk/NGBOViewGrant.aspx?GrantRef=EP/N027221/1. [Accessed: 02-Aug-2017]. 